Research Article

\title{
Super-Twisting Sliding Mode Control Law Design for Attitude Tracking Task of a Spacecraft via Reaction Wheels
}

\author{
Yang-Rui Li iD and Chao-Chung Peng (iD \\ Department of Aeronautics and Astronautics, National Cheng Kung University, Tainan, Taiwan \\ Correspondence should be addressed to Chao-Chung Peng; ccpeng@mail.ncku.edu.tw
}

Received 3 October 2020; Revised 19 November 2020; Accepted 8 December 2020; Published 16 March 2021

Academic Editor: Haibo Du

Copyright (C) 2021 Yang-Rui Li and Chao-Chung Peng. This is an open access article distributed under the Creative Commons Attribution License, which permits unrestricted use, distribution, and reproduction in any medium, provided the original work is properly cited.

\begin{abstract}
The attitude control has been recognized as one of the most important research topics for spacecraft. If the desired attitude trajectory cannot be tracked precisely, it may cause mission failures. In the real space mission environment, the unknown external perturbations, for example, atmospheric drag and solar radiation, should be taken into consideration. Such external perturbations could deviate the precision of the spacecraft orientation and thereby lead to a mission failure. Therefore, in this paper, a quaternion-based super-twisting sliding mode robust control law for the spacecraft attitude tracking is developed. The finite time stability based on the formulation of the linear matrix inequality (LMI) is also provided. To avoid losing the control degree of freedom due to the certain actuator fault, a redundant reaction wheels configuration is adopted. The actuators distribution associated force distribution matrix (FDM) is analyzed in detail. Finally, the reference tangent-normal-binormal (TNB) command generation strategy is implemented for simulating the scenario of the space mission. Finally, the simulation results reveal that the spacecraft can achieve the desired attitude trajectory tracking demands in the presence of the time-varying external disturbances.
\end{abstract}

\section{Introduction}

Sliding mode control (SMC) techniques have been a popular research topic of the control theory in recent years such as adaptive super-twisting SMC [1-3], fractional-order sliding mode control [4], finite time control [5], robust backstepping SMC [6-8], and model predictive SMC [9]. The superior robustness to the matched perturbations is one of the features of the SMC. However, the price of the robustness is the chattering effect of the control signal. It causes application difficulty for practical implementations [10]. The ways to attenuate the chattering phenomenon include the following [11-13]: (i) replacing the discontinuous switching function with a saturation function or a sigmoid function, (ii) applying an adaptive law to adjust the switching gain dynamically, and (iii) using the higher-order SMC techniques. Nevertheless, skill (i) results in losing the robustness to the disturbances. Even though approach (ii) can estimate an adequate magnitude of the switching gain with respect to perturbations $[14,15]$, the estimation of the switch gain could increase monotonically due to the absence of perfect sliding motion in practice. For (iii), the gain/ stability determinations are quite challenging.

The high-order SMC approach can drive the sliding variable and its consecutive derivations to zero in the presence of the matched perturbations. However, the main challenge of the high-order SMC is that it uses the information of the high-order time derivatives of the sliding variable [16-18]. Among the higher-order SMC techniques, it is worth remarking that the second-order SMC such as the super-twisting algorithm only needs the feedback information of the sliding variable in control process. The supertwisting algorithm was firstly proposed by Dr. Levant in 1993 [19]. A quadratic Lyapunov function proposed in [20] is considered in the proof of the finite-time convergence property. The successive researches include [21-24]. Owing to the superior properties, the super-twisting algorithm has been applied in several studies, including quadrotor $[25,26]$, industrial emulator [27], and mobile wheeled inverted pendulum [28]. For this reason, the robust continuous 
super-twisting sliding mode algorithm will be adopted for the attitude tracking control design in this paper.

The reaction wheel driven based system is actuated by means of generating the reaction torque from the wheel. The reaction wheel has been widely applied in the most dynamics systems. In literature [29-31], the reaction wheels are used in the attitude tracking control demands. The works in [32, 33] use the reaction wheel to address the balancing control of the inverted pendulum. The main objective of this paper is to apply the super-twisting algorithm for the attitude control of the spacecraft via using four reaction wheels as the actuated source. The attitude representation includes several approaches, eor example, Euler angles, Rodrigues parameters, and quaternion [34]. To avoid singularity, the quaternionbased control is considered. The quaternion-based control has been proposed in several studies $[30,35,36]$. However, it assumes that the scalar component of error quaternion $q_{0 e}$ does not equal zero to guarantee that the matrix $0.5\left(q_{0 e} \mathbf{I}_{3}+\right.$ $\left.\mathbf{q}_{e}^{\times}\right)$is invertible. This assumption leads to the controllers containing a singularity when $q_{0 e}=0$. It should be noted that one of the reasons to use quaternion-based control is to obtain the full attitude tracking task and avoid any singularity limitations. As a result, in this paper, a quaternionbased super-twisting sliding mode algorithm is adopted in the controller design such that the robust performance can be guaranteed. The asymptotic stability proof of the nonlinear reduced-order dynamics by means of an analytic solution will be addressed without imposing assumptions.

Regarding the organization of this article, in Section 2, the governing equations of attitude dynamics based on the quaternion kinematics and the redundant reaction wheels configurations are derived. The configuration is introduced from $[30,37,38]$. To obtain the feasible reaction torques, the FDM is reformulated as a square and invertible matrix, which minimizes the control energy cost [30]. In Section 3, a robust, continuous super-twisting sliding mode algorithm is considered in the controller design so that the spacecraft can handle the external perturbations in the real and complex space environment. The stability problem will be reformulated as a feasibility problem of a LMI and therefore the finite time stability can be achieved in the sense of Lyapunov. In Section 4, the reference TNB command generation strategy is proposed to verify the tracking performance of the spacecraft. In Section 5, the numerical simulation is carried out and the results reveal that the spacecraft can track the desired attitude trajectory in the presence of time-varying disturbances.

The contributions of this paper are summarized as follows: (i) realizes the super-twisting sliding mode algorithm as a robust, continuous quaternion-based attitude controller for the attitude trajectory tracking demands of a spacecraft with the redundant reaction wheels; (ii) proposes a modified version of LMI which has higher degrees of freedom for finding the decision variables and it can satisfy the convergence performance by requirement; (iii) derives the analytic solution of the nonlinear reduced-order dynamics; and (iv) presents a reference TNB command generation strategy so that the feasibility of the controller can be verified.

\section{Reaction Wheels Driven Based on Spacecraft Dynamics Modeling}

2.1. Geometry Configuration Analysis. From the perspective of practical realization, to avoid losing a degree of freedom of control in space due to certain actuator faults, the redundant reaction wheels configuration is adopted $[30,37,38]$. The dynamic configuration is shown in Figure 1. To formally derive the governing equations of the attitude dynamics, we firstly define the coordinate system as follows: (i) the body frame denoted as $x y z$, which is fixed in the body of the spacecraft to represent the attitude of the spacecraft, and (ii) the auxiliary rotation frame denoted as $x_{i} y_{i} z_{i}$, which is fixed in the $i$-th reaction wheel to describe the relative rotation of $i$-th reaction wheel to spacecraft.

Taking the first reaction wheel as an example, the geometry mapping relation between $x y z$ and $x_{1} y_{1} z_{1}$ is explained in Figures 2 and 3, respectively. Referring to Figure 2, the body frame $x y z$ rotates about the $z$-axis with an angle $\beta$, and the new frame is denoted as $x^{\prime} y^{\prime} z^{\prime}$. From the rotation property, the mapping relation between $x^{\prime} y^{\prime} z^{\prime}$ and $x y z$ can be constructed as

$$
\left[\begin{array}{l}
x^{\prime} \\
y^{\prime} \\
z^{\prime}
\end{array}\right]=\left[\begin{array}{ccc}
\cos \beta & \sin \beta & 0 \\
-\sin \beta & \cos \beta & 0 \\
0 & 0 & 1
\end{array}\right]\left[\begin{array}{l}
x \\
y \\
z
\end{array}\right] .
$$

Regarding Figure 3, the frame $x^{\prime} y^{\prime} z^{\prime}$ rotates about negative $y$-axis with an angle $\alpha$, and then the new frame $x^{\prime \prime} y^{\prime \prime} z^{\prime \prime}$ is obtained. Again, from the rotation property, we have the mapping relation between $x^{\prime} y^{\prime} z^{\prime}$ and $x^{\prime \prime} y^{\prime \prime} z^{\prime \prime}$ :

$$
\left[\begin{array}{l}
x^{\prime \prime} \\
y^{\prime \prime} \\
z^{\prime \prime}
\end{array}\right]=\left[\begin{array}{ccc}
\cos (-\alpha) & 0 & -\sin (-\alpha) \\
0 & 1 & 0 \\
\sin (-\alpha) & 0 & \cos (-\alpha)
\end{array}\right]\left[\begin{array}{l}
x^{\prime} \\
y^{\prime} \\
z^{\prime}
\end{array}\right] .
$$

On the basis of previous illustrations, the mapping relation between $x y z$ and $x_{1} y_{1} z_{1}$ can be derived by combining (1) and (2):

$$
\begin{aligned}
{\left[\begin{array}{l}
x_{1} \\
y_{1} \\
z_{1}
\end{array}\right]=} & {\left[\begin{array}{l}
x^{\prime \prime} \\
y^{\prime \prime} \\
z^{\prime \prime}
\end{array}\right]=\left[\begin{array}{ccc}
\cos (-\alpha) & 0 & -\sin (-\alpha) \\
0 & 1 & 0 \\
\sin (-\alpha) & 0 & \cos (-\alpha)
\end{array}\right]\left[\begin{array}{l}
x^{\prime} \\
y^{\prime} \\
z^{\prime}
\end{array}\right] } \\
= & {\left[\begin{array}{ccc}
\cos (-\alpha) & 0 & -\sin (-\alpha) \\
0 & 1 & 0 \\
\sin (-\alpha) & 0 & \cos (-\alpha)
\end{array}\right]\left[\begin{array}{ccc}
\cos \beta & \sin \beta & 0 \\
-\sin \beta & \cos \beta & 0 \\
0 & 0 & 1
\end{array}\right]\left[\begin{array}{l}
x \\
y \\
z
\end{array}\right] . }
\end{aligned}
$$

In general, the mapping relation between $x y z$ and $x_{i} y_{i} z_{i}$ can be formulated as

$$
\left[\begin{array}{c}
x_{i} \\
y_{i} \\
z_{i}
\end{array}\right]=\mathbf{R}_{i}\left[\begin{array}{l}
x \\
y \\
z
\end{array}\right]
$$

where 


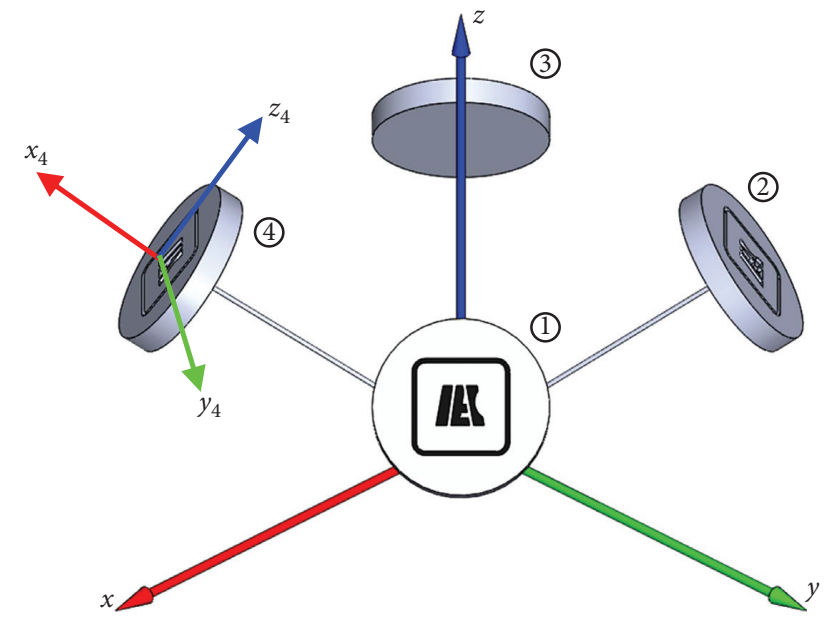

FIGURE 1: Geometry prototype of the redundant reaction wheels configuration.

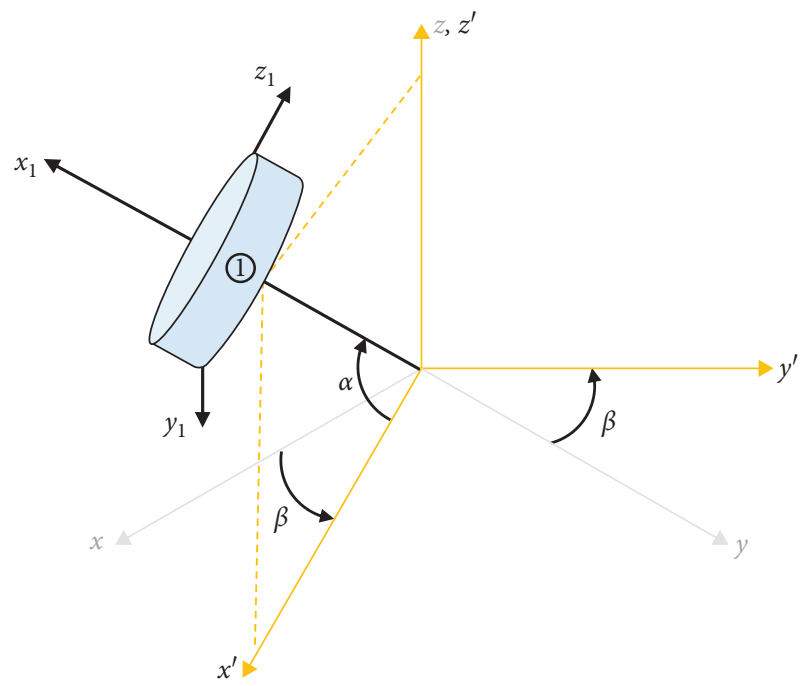

Figure 2: Rotation of body frame $x y z$ to frame $x^{\prime} y^{\prime} z^{\prime}$.

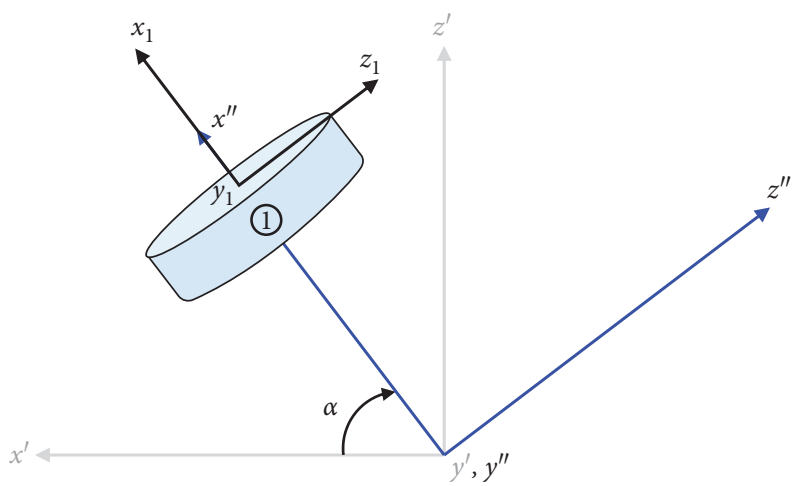

Figure 3: Rotation of frame $x^{\prime} y^{\prime} z^{\prime}$ to frame $x^{\prime \prime} y^{\prime \prime} z^{\prime \prime}$.

$$
\begin{aligned}
\mathbf{R}_{i} & =\left[\begin{array}{ccc}
\cos (-\alpha) & 0 & -\sin (-\alpha) \\
0 & 1 & 0 \\
\sin (-\alpha) & 0 & \cos (-\alpha)
\end{array}\right]\left[\begin{array}{ccc}
\cos \beta_{i} & \sin \beta_{i} & 0 \\
-\sin \beta_{i} & \cos \beta_{i} & 0 \\
0 & 0 & 1
\end{array}\right] \\
& =\left[\begin{array}{ccc}
\cos \alpha \cos \beta_{i} & \cos \alpha \sin \beta_{i} & \sin \alpha \\
-\sin \beta_{i} & \cos \beta_{i} & 0 \\
-\cos \beta_{i} \sin \alpha & -\sin \alpha \sin \beta_{i} & \cos \alpha
\end{array}\right],
\end{aligned}
$$

and $\beta_{i}=\beta+0.5(i-1) \pi, i=1,2,3,4$.

2.2. Attitude Dynamics. To avoid the singularity problem in the Euler approaches, the quaternion-based attitude representation is considered. Let $\mathbf{Q}=\left[q_{0}, \mathbf{q}^{T}\right]^{T}=\left[q_{0}\right.$, $\left.q_{1}, q_{2}, q_{3}\right]^{T} \in \mathbb{R}^{4}$ be the unit quaternion and let $\boldsymbol{\omega}=\left[\omega_{x}, \omega_{y}, \omega_{z}\right]^{T} \in \mathbb{R}^{3}$ be the angular velocity of spacecraft; then the quaternion kinematic equations are given by

$$
\begin{aligned}
\dot{q}_{0} & =-\frac{1}{2} \mathbf{q}^{T} \boldsymbol{\omega}, \\
\dot{\mathbf{q}} & =\frac{1}{2}\left(q_{0} \mathbf{I}_{3}+\mathbf{q}^{\times}\right) \boldsymbol{\omega} .
\end{aligned}
$$

The above equations can be rewritten in a compact matrix form:

$$
\dot{\mathbf{Q}}=\frac{1}{2} \mathbf{E}(\mathbf{Q}) \boldsymbol{\omega},
$$

where

$$
\mathbf{E}(\mathbf{Q})=\left[\begin{array}{c}
-\mathbf{q}^{T} \\
q_{0} \mathbf{I}_{3}+\mathbf{q}^{\times}
\end{array}\right]=\left[\begin{array}{ccc}
-q_{1} & -q_{2} & -q_{3} \\
q_{0} & -q_{3} & q_{2} \\
q_{3} & q_{0} & -q_{1} \\
-q_{2} & q_{1} & q_{0}
\end{array}\right] .
$$

The symbol $\mathbf{a}^{\times}$represents a cross product matrix of vector $\mathbf{a}=\left[a_{1}, a_{2}, a_{3}\right]^{T} \in \mathbb{R}^{3}$, which is defined as

$$
\mathbf{a}^{\times}=\left[\begin{array}{ccc}
0 & -a_{3} & a_{2} \\
a_{3} & 0 & -a_{1} \\
-a_{2} & a_{1} & 0
\end{array}\right] .
$$

In the following, the kinetic equations based on the redundant reaction wheels configuration will be derived. Let $\Omega_{i}, i=1,2,3,4$, be the relative rotational speed of each reaction wheel to the spacecraft; then the angular velocity of each reaction wheel with respect to $x_{i} y_{i} z_{i}$ is given by

$$
\begin{aligned}
\boldsymbol{\omega}_{w, i} & =\mathbf{R}_{i} \boldsymbol{\omega}+\Omega_{i} \mathbf{e}_{x_{i}}, \\
\mathbf{e}_{x_{i}} & =\left[\begin{array}{lll}
1 & 0 & 0
\end{array}\right]^{T} .
\end{aligned}
$$

The angular momentum of spacecraft relative the body frame $x y z$ is 


$$
\mathbf{H}=\mathbf{J} \boldsymbol{\omega}=\left[\begin{array}{ccc}
J_{x} & -J_{x y} & -J_{x z} \\
-J_{x y} & J_{y} & -J_{y z} \\
-J_{x z} & -J_{y z} & J_{z}
\end{array}\right]\left[\begin{array}{c}
\omega_{x} \\
\omega_{y} \\
\omega_{z}
\end{array}\right] .
$$

Assume that the reaction wheels are perfect circle plate. Hence, the moment of inertia matrix of $i$-th reaction wheel with respect to $x_{i} y_{i} z_{i}$ is

$$
\mathbf{J}_{w, i}=\left[\begin{array}{ccc}
J_{m, i} & 0 & 0 \\
0 & J_{i}^{\prime} & 0 \\
0 & 0 & J_{i}^{\prime}
\end{array}\right] .
$$

The angular momentum of each reaction wheel relative frame $x_{i} y_{i} z_{i}$ can be constructed:

$$
\begin{aligned}
\mathbf{H}_{w, i} & =\mathbf{J}_{w, i} \boldsymbol{\omega}_{w, i} \\
& =\mathbf{J}_{w, i}\left(\mathbf{R}_{i} \boldsymbol{\omega}+\Omega_{i} \mathbf{e}_{x_{i}}\right) \\
& =\mathbf{J}_{w, i} \mathbf{R}_{i} \boldsymbol{\omega}+\mathbf{J}_{w} \Omega_{i} \mathbf{e}_{x_{i}} \\
& =\mathbf{J}_{w, i} \mathbf{R}_{i} \boldsymbol{\omega}+J_{m i} \Omega_{i} \mathbf{e}_{x_{i}} .
\end{aligned}
$$

Mapping $\mathbf{H}_{w, i}$ onto spacecraft body frame $x y z$ yields

$$
\begin{aligned}
\mathbf{H}_{w, i}^{B} & =\mathbf{R}_{i}^{T} \boldsymbol{\omega}_{w, i} \\
& =\mathbf{R}_{i}^{T}\left(\mathbf{J}_{w, i} \mathbf{R}_{i} \boldsymbol{\omega}+J_{m i} \Omega_{i} \mathbf{e}_{x_{i}}\right) \\
& =\mathbf{R}_{i}^{T} \mathbf{J}_{w, i} \mathbf{R}_{i} \boldsymbol{\omega}+\mathbf{R}_{i}^{T} J_{m i} \Omega_{i} \mathbf{e}_{x_{i}} .
\end{aligned}
$$

The total angular momentum of the system (spacecraft and reaction wheels), $\mathbf{H}_{T}$, is the summation of $\mathbf{H}$ and $\mathbf{H}_{w, i}^{B}$, which is

$$
\begin{aligned}
\mathbf{H}_{T} & =\mathbf{H}+\sum_{i=1}^{4} \mathbf{H}_{w, i}^{B} \\
& =\mathbf{J} \boldsymbol{\omega}+\sum_{i=1}^{4}\left(\mathbf{R}_{i}^{T} \mathbf{J}_{w, i} \mathbf{R}_{i} \boldsymbol{\omega}+\mathbf{R}_{i}^{T} J_{m i} \Omega_{i} \mathbf{e}_{x_{i}}\right) \\
& =\left(\mathbf{J}+\sum_{i=1}^{4} \mathbf{R}_{i}^{T} \mathbf{J}_{w, i} \mathbf{R}_{i}\right) \boldsymbol{\omega}+\sum_{i=1}^{4} \mathbf{R}_{i}^{T} J_{m i} \Omega_{i} \mathbf{e}_{x_{i}} .
\end{aligned}
$$

The term

$$
\begin{aligned}
\sum_{i=1}^{4} \mathbf{R}_{i}^{T} J_{m} \Omega_{i} \mathbf{e}_{x_{i}} & =\left[\begin{array}{c}
\cos \alpha \cos \beta \\
\cos \alpha \sin \beta \\
\sin \alpha
\end{array}\right] J_{m 1} \Omega_{1}+\left[\begin{array}{c}
-\cos \alpha \sin \beta \\
\cos \alpha \cos \beta \\
\sin \alpha
\end{array}\right] J_{m 2} \Omega_{2}+\left[\begin{array}{c}
-\cos \alpha \cos \beta \\
-\cos \alpha \sin \beta \\
\sin \alpha
\end{array}\right] J_{m 3} \Omega_{3}+\left[\begin{array}{c}
\cos \alpha \cos \beta \\
-\cos \alpha \sin \beta \\
\sin \alpha
\end{array}\right] J_{m 4} \Omega_{4} \\
& =\left[\begin{array}{ccccc}
\cos \alpha \cos \beta & -\cos \alpha \sin \beta & -\cos \alpha \cos \beta & \cos \alpha \sin \beta \\
\cos \alpha \sin \beta & \cos \alpha \cos \beta & -\cos \alpha \sin \beta & -\cos \alpha \cos \beta \\
\sin \alpha & \sin \alpha & \sin \alpha & \sin \alpha
\end{array}\right]\left[\begin{array}{cccc}
J_{m 1} & 0 & 0 & 0 \\
0 & J_{m 2} & 0 & 0 \\
0 & 0 & J_{m 3} & 0 \\
0 & 0 & 0 & J_{m 4}
\end{array}\right]\left[\begin{array}{l}
\Omega_{1} \\
\Omega_{2} \\
\Omega_{3} \\
\Omega_{4}
\end{array}\right]
\end{aligned}
$$

Define the following:

(i) Equivalent moment of inertia matrix of the system:

$$
\mathbf{J}_{e q}=\mathbf{J}+\sum_{i=1}^{4} \mathbf{R}_{i}^{T} \mathbf{J}_{w, i} \mathbf{R}_{i} .
$$

(ii) Force distribution matrix (FDM):

$$
\Gamma=\left[\begin{array}{cccc}
\cos \alpha \cos \beta & -\cos \alpha \sin \beta & -\cos \alpha \cos \beta & \cos \alpha \sin \beta \\
\cos \alpha \sin \beta & \cos \alpha \cos \beta & -\cos \alpha \sin \beta & -\cos \alpha \cos \beta \\
\sin \alpha & \sin \alpha & \sin \alpha & \sin \alpha
\end{array}\right] .
$$

(iii) Axial moment of inertia matrix:

$$
\mathbf{J}_{m}=\left[\begin{array}{cccc}
J_{m 1} & 0 & 0 & 0 \\
0 & J_{m 2} & 0 & 0 \\
0 & 0 & J_{m 3} & 0 \\
0 & 0 & 0 & J_{m 4}
\end{array}\right] .
$$

Combining (17)-(20), equation (16) can be further simplified to

$$
\mathbf{H}_{T}=\mathbf{J}_{e q} \boldsymbol{\omega}+\Gamma \mathbf{J}_{m} \mathbf{\Omega},
$$

where $\Omega=\left[\Omega_{1}, \Omega_{2}, \Omega_{3}, \Omega_{4}\right]^{T}$. The Euler equation of motion is given by

$$
\mathbf{M}_{G}=\left(\frac{\mathrm{d} \mathbf{H}_{T}}{\mathrm{~d} t}\right)_{B}+\boldsymbol{\omega}^{\times} \mathbf{H}_{T},
$$

in which the total external torque $\mathbf{M}_{G}$ is equal to the summation of the external control torques $\tau_{a}$ and the external disturbance torques $\mathbf{d}(t)$. Substituting (21) into (22) yields

$$
\boldsymbol{\tau}_{a}+\mathbf{d}=\mathbf{J}_{e q} \dot{\boldsymbol{\omega}}+\Gamma \mathbf{J}_{m} \dot{\boldsymbol{\Omega}}+\boldsymbol{\omega}^{\times}\left(\mathbf{J}_{e q} \boldsymbol{\omega}+\Gamma \mathbf{J}_{m} \boldsymbol{\Omega}\right) .
$$

Define the control torque $\tau$ and the reaction torque $\tau_{w}$ as

$$
\begin{aligned}
& \boldsymbol{\tau}_{c}=\Gamma \tau_{w} \triangleq\left[\begin{array}{lll}
\tau_{c x} & \tau_{c y} & \tau_{c z}
\end{array}\right]^{T}, \\
& \boldsymbol{\tau}_{w}=-\mathbf{J}_{m} \dot{\boldsymbol{\Omega}}=-\left[\begin{array}{llllll}
J_{m 1} \dot{\Omega}_{1} & J_{m 2} \dot{\Omega}_{2} & J_{m 3} \dot{\Omega}_{3} & J_{m 4} \dot{\Omega}_{4}
\end{array}\right]^{T} \\
& \triangleq\left[\begin{array}{llll}
\tau_{w 1} & \tau_{w 2} & \tau_{w 3} & \tau_{w 4}
\end{array}\right]^{T} .
\end{aligned}
$$


Hence, (23) can be further simplified as

$$
\mathbf{J}_{e q} \dot{\boldsymbol{\omega}}=\boldsymbol{\tau}_{a}+\boldsymbol{\tau}_{c}+\mathbf{d}-\boldsymbol{\omega}^{\times}\left(\mathbf{J}_{e q} \boldsymbol{\omega}+\Gamma \mathbf{J}_{m} \boldsymbol{\Omega}\right) .
$$

In this paper, $\boldsymbol{\tau}_{a}=\mathbf{0}$ is considered.

\subsection{Actuator Analysis. From (24), we have}

$$
\left[\begin{array}{c}
\tau_{c x} \\
\tau_{c y} \\
\tau_{c z}
\end{array}\right]=\left[\begin{array}{cccc}
\cos \alpha \cos \beta & -\cos \alpha \sin \beta & -\cos \alpha \cos \beta & \cos \alpha \sin \beta \\
\cos \alpha \sin \beta & \cos \alpha \cos \beta & -\cos \alpha \sin \beta & -\cos \alpha \cos \beta \\
\sin \alpha & \sin \alpha & \sin \alpha & \sin \alpha
\end{array}\right]\left[\begin{array}{c}
\tau_{w 1} \\
\tau_{w 2} \\
\tau_{w 3} \\
\tau_{w 4}
\end{array}\right] .
$$

Once $\tau_{c}$ is designed for the attitude trajectory tracking demands of the system dynamics (6), (7), and (25), it is desired to obtain each reaction torque $\tau_{w i}$. However, FDM (19) is a nonsquare matrix, so the inverse does not exist. To obtain the FDM with a special matrix structure, the two following geometry constraints are imposed [30]:

$$
\begin{array}{r}
\sin \beta=\cos \beta, \quad 0 \leq \beta<\frac{\pi}{2}, \\
\cos \alpha \sin \beta=\sin \alpha, \quad 0 \leq \alpha<\frac{\pi}{2},
\end{array}
$$

or, equivalently,

$$
\begin{aligned}
\beta & =\frac{\pi}{4}, \\
\sin \alpha & =\frac{\sqrt{3}}{3} .
\end{aligned}
$$

From (27), we have the following FDM:

$$
\Gamma=\frac{\sqrt{3}}{3}\left[\begin{array}{cccc}
1 & -1 & -1 & 1 \\
1 & 1 & -1 & -1 \\
1 & 1 & 1 & 1
\end{array}\right]
$$

and then the following static optimization problem is formulated [30]:

$$
\min _{\tau_{w}} \mathscr{T}=\sum_{i=1}^{4} \tau_{w i}^{2}
$$

subject to

$$
\begin{aligned}
& g_{1} \triangleq \frac{\sqrt{3}}{3}\left(\tau_{w 1}-\tau_{w 2}-\tau_{w 3}+\tau_{w 4}\right)-\tau_{c x}=0 \\
& g_{2} \triangleq \frac{\sqrt{3}}{3}\left(\tau_{w 1}+\tau_{w 2}-\tau_{w 3}-\tau_{w 4}\right)-\tau_{c y}=0 \\
& g_{3} \triangleq \frac{\sqrt{3}}{3}\left(\tau_{w 1}+\tau_{w 2}+\tau_{w 3}+\tau_{w 4}\right)-\tau_{c z}=0
\end{aligned}
$$

To formally address the problem, refer to [39], and define the Lagrangian $\mathscr{L}$ together with the Lagrange multiplier $\lambda=$ $\left[\boldsymbol{\lambda}_{1}, \boldsymbol{\lambda}_{2}, \boldsymbol{\lambda}_{3}\right]^{T} \in \mathbb{R}^{3}$ as follows:

$$
\mathscr{L}=\mathscr{T}+\lambda_{1} g_{1}+\lambda_{2} g_{2}+\lambda_{3} g_{3} .
$$

Let $\boldsymbol{\tau}_{w}^{*}$ and $\lambda^{*}$ be the optimal solution. The first-order necessary condition to minimize $\mathscr{T}$ is

$$
\begin{aligned}
& \left.\frac{\partial \mathscr{L}}{\partial \tau_{w 1}}\right|_{\tau^{*}, \lambda^{*}}=2 \tau_{w 1}^{*}+\frac{\sqrt{3}}{3} \lambda_{1}^{*}+\frac{\sqrt{3}}{3} \lambda_{2}^{*}+\frac{\sqrt{3}}{3} \lambda_{3}^{*}=0, \\
& \left.\frac{\partial \mathscr{L}}{\partial \tau_{w 2}}\right|_{\tau^{*}, \lambda^{*}}=2 \tau_{w 2}^{*}-\frac{\sqrt{3}}{3} \lambda_{1}^{*}+\frac{\sqrt{3}}{3} \lambda_{2}^{*}+\frac{\sqrt{3}}{3} \lambda_{3}^{*}=0, \\
& \left.\frac{\partial \mathscr{L}}{\partial \tau_{w 3}}\right|_{\tau^{*}, \lambda^{*}}=2 \tau_{w 3}^{*}-\frac{\sqrt{3}}{3} \lambda_{1}^{*}-\frac{\sqrt{3}}{3} \lambda_{2}^{*}+\frac{\sqrt{3}}{3} \lambda_{3}^{*}=0, \\
& \left.\frac{\partial \mathscr{L}}{\partial \tau_{w 4}}\right|_{\tau^{*}, \lambda^{*}}=2 \tau_{w 4}^{*}+\frac{\sqrt{3}}{3} \lambda_{1}^{*}-\frac{\sqrt{3}}{3} \lambda_{2}^{*}+\frac{\sqrt{3}}{3} \lambda_{3}^{*}=0,
\end{aligned}
$$

and

$$
\begin{aligned}
& \left.\frac{\partial \mathscr{L}}{\partial \lambda_{1}}\right|_{\tau^{*}, \lambda^{*}}=\frac{\sqrt{3}}{3}\left(\tau_{w 1}^{*}-\tau_{w 2}^{*}-\tau_{w 3}^{*}+\tau_{w 4}^{*}\right)-\tau_{c x}=0, \\
& \left.\frac{\partial \mathscr{L}}{\partial \lambda_{2}}\right|_{\tau^{*}, \lambda^{*}}=\frac{\sqrt{3}}{3}\left(\tau_{w 1}^{*}+\tau_{w 2}^{*}-\tau_{w 3}^{*}-\tau_{w 4}^{*}\right)-\tau_{c y}=0, \\
& \left.\frac{\partial \mathscr{L}}{\partial \lambda_{3}}\right|_{\tau^{*}, \lambda^{*}}=\frac{\sqrt{3}}{3}\left(\tau_{w 1}^{*}+\tau_{w 2}^{*}+\tau_{w 3}^{*}+\tau_{w 4}^{*}\right)-\tau_{c z}=0 .
\end{aligned}
$$

From (33), it is implied that

$$
\tau_{w 1}^{*}-\tau_{w 2}^{*}+\tau_{w 3}^{*}-\tau_{w 4}^{*}=0 .
$$

Combining (34) and (35), the distribution matrix can be augmented as

$$
\left[\begin{array}{c}
\tau_{c x} \\
\tau_{c y} \\
\tau_{c z} \\
0
\end{array}\right]=\left[\begin{array}{cccc}
\frac{\sqrt{3}}{3} & -\frac{\sqrt{3}}{3} & -\frac{\sqrt{3}}{3} & \frac{\sqrt{3}}{3} \\
\frac{\sqrt{3}}{3} & \frac{\sqrt{3}}{3} & -\frac{\sqrt{3}}{3} & -\frac{\sqrt{3}}{3} \\
\frac{\sqrt{3}}{3} & \frac{\sqrt{3}}{3} & \frac{\sqrt{3}}{3} & \frac{\sqrt{3}}{3} \\
1 & -1 & 1 & -1
\end{array}\right]\left[\begin{array}{c}
\tau_{w 1}^{*} \\
\tau_{w 2}^{*} \\
\tau_{w 3}^{*} \\
\tau_{w 4}^{*}
\end{array}\right] .
$$

The inverse mapping is

$$
\left[\begin{array}{c}
\tau_{w 1}^{*} \\
\tau_{w 2}^{*} \\
\tau_{w 3}^{*} \\
\tau_{w 4}^{*}
\end{array}\right]=\frac{1}{4}\left[\begin{array}{cccc}
\sqrt{3} & \sqrt{3} & \sqrt{3} & 1 \\
-\sqrt{3} & \sqrt{3} & \sqrt{3} & -1 \\
-\sqrt{3} & -\sqrt{3} & \sqrt{3} & 1 \\
\sqrt{3} & -\sqrt{3} & \sqrt{3} & -1
\end{array}\right]\left[\begin{array}{c}
\tau_{c x} \\
\tau_{c y} \\
\tau_{c z} \\
0
\end{array}\right] .
$$

Hence, when $\tau_{c}$ is designed for the system dynamics (6), (7), and (25), the reaction torque $\tau_{w i}$ for each reaction wheel can be obtained by inverse mapping (37). Moreover, it can be guaranteed that the reaction torque is an optimal value $\tau_{w}^{*}$ to minimize the performance index (30). 
Because the reaction wheels are actuated by servo motors, that is, the reaction torques are generated by servo motors, the following linear dynamic equation is considered:

$$
J_{m i} \dot{\Omega}_{i}=-a_{i} \Omega_{i}+b_{i} V_{\mathrm{in}, i}, \quad i=1,2,3,4 .
$$

From the definition, (24) and (38) become

$$
V_{\mathrm{in}, i}=\frac{1}{b_{i}}\left(-\tau_{w i}+a_{i} \Omega_{i}\right) \quad i=1,2,3,4,
$$

where the parameters $a_{i}=0.02$ and $b_{i}=3$ are considered. $V_{\mathrm{in}, i}$ is the control input voltage.

Conclusively, $\boldsymbol{\tau}_{c}$ is first designed for the system dynamics (6), (7), and (25). Secondly, inverse mapping (37) is used to obtain the optimal reaction torque $\tau_{w i}$ of each reaction wheel to minimize the energy cost (30). Finally, apply (39) to obtain the corresponding control voltage $V_{\mathrm{in}, i}$. In the following, the discussion focuses on how to design $\boldsymbol{\tau}_{c}$ so that the desired trajectory can be achieved in the presence of timevarying disturbances.

\section{Super-Twisting Sliding Mode Controller Design}

3.1. Super-Twisting Sliding Mode Algorithm. The design process of sliding mode control includes two steps: (i) A sliding variable $s$ is designed so that the stability of the reduced-order dynamics can be guaranteed. (ii) Seeking the robust, continuous control law to guarantee the sliding mode $s=\dot{s}=0$ occurs in a finite time in the presence of the time-varying external disturbances. In the following, the super-twisting sliding mode algorithm [19] is introduced.
The super-twisting sliding mode algorithm is one of the outstanding robust control algorithms which handles a system with a relative degree $(u \longrightarrow s)$ equal to one. Based on the algorithm, the closed-loop sliding dynamics is designed as

$$
\dot{s}=-k_{1}|s|^{1 / 2} \operatorname{sign}(s)-k_{2} \int_{0}^{t} \operatorname{sign}(s) \mathrm{d} \tau+\mathrm{d}(t),
$$

where $s=s(\mathrm{x}, t) \in \mathbb{R}$ is the sliding variable; $\mathrm{x} \in \mathbb{R}^{n}$ is state vector; $\mathrm{d}(t) \in \mathbb{R}$ is an unknown time-varying perturbation and it is assumed that $|\dot{\mathrm{d}}(t)| \leq \delta$; the gain pair $\left(k_{1}, k_{2}\right)$ is to be designed so that the sliding mode can occur in a finite time.

In fact, the more general representation of (40) is to express it as the following state-space form. Let

$$
\begin{aligned}
& z_{1}=s(x, t) . \\
& z_{2}=-k_{2} \int_{0}^{t} \operatorname{sign}(s) \mathrm{d} \tau+\mathrm{d}(t),
\end{aligned}
$$

which implies

$$
\begin{aligned}
& \dot{z}_{1}=-k_{1}\left|z_{1}\right|^{1 / 2} \operatorname{sign}\left(z_{1}\right)+z_{2}, \\
& \dot{z}_{2}=-k_{2} \operatorname{sign}\left(z_{1}\right)+\rho,
\end{aligned}
$$

where $\rho(t)=\dot{d}(t)$ and $|\rho(t)|=|\dot{d}(t)| \leq \delta$. Since (42) is nonlinear, consider the following variable transformation [20]:

$$
\begin{aligned}
& \zeta_{1}=\left|z_{1}\right|^{1 / 2} \operatorname{sign}\left(z_{1}\right), \\
& \zeta_{2}=z_{2} .
\end{aligned}
$$

Taking the time derivative yields

$$
\begin{aligned}
\dot{\zeta}_{1} & =\frac{\dot{z}_{1}\left|z_{1}\right|^{1 / 2}-z_{1} \cdot(1 / 2)\left|z_{1}\right|^{-1 / 2} \cdot \operatorname{sign}\left(z_{1}\right) \cdot \dot{z}_{1}}{\left|z_{1}\right|}=\frac{\left[-k_{1}\left|z_{1}\right|^{1 / 2} \operatorname{sign}\left(z_{1}\right)+z_{2}\right] \cdot\left(\left|z_{1}\right|^{1 / 2}-(1 / 2)\left|z_{1}\right|^{1 / 2}\right)}{\left|z_{1}\right|} \\
& =\frac{1}{\left|z_{1}\right|^{1 / 2}}\left(-\frac{k_{1}}{2}\left|z_{1}\right|^{1 / 2} \operatorname{sign}\left(z_{1}\right)+\frac{1}{2} z_{2}\right)=\frac{1}{\left|\zeta_{1}\right|}\left(-\frac{k_{1}}{2} \zeta_{1}+\frac{1}{2} \zeta_{2}\right), \\
\dot{\zeta}_{2} & =-k_{2} \operatorname{sign}\left(z_{1}\right)+\rho=\frac{1}{\left|z_{1}\right|^{1 / 2}}\left(-k_{2}\left|z_{1}\right|^{1 / 2} \operatorname{sign}\left(z_{1}\right)+\left|z_{1}\right|^{1 / 2} \rho\right)=\frac{1}{\left|\zeta_{1}\right|}\left(-k_{2} \zeta_{1}+\left|\zeta_{1}\right| \rho\right),
\end{aligned}
$$

which can be rewritten in the matrix form as

$$
\begin{aligned}
& \dot{\zeta}=\frac{1}{\left|\zeta_{1}\right|}(\mathbf{A} \zeta+\mathbf{B} \widetilde{\rho}), \\
& \mathbf{A}=\left[\begin{array}{cc}
-0.5 k_{1} & 0.5 \\
-k_{2} & 0
\end{array}\right], \\
& \mathbf{B}=\left[\begin{array}{l}
0 \\
1
\end{array}\right],
\end{aligned}
$$

where $\zeta=\left[\zeta_{1}, \zeta_{2}\right]^{T}$ and $\left|\zeta_{1}\right|=\left|z_{1}\right|^{1 / 2}$. Disturbance transformation $\tilde{\rho}\left(t, \zeta_{1}\right)=\rho(t)\left|\zeta_{1}\right|$ satisfies

$$
\left|\widetilde{\rho}\left(t, \zeta_{1}\right)\right| \leq \delta\left|\zeta_{1}\right| .
$$

For system (45), the stability problem is proven in the following. It is shown that the stability issue can be reformulated as a feasibility problem in terms of the LMI.

Theorem 1 (See[21, 22, 25]). Suppose that there exist symmetric and positive definite matrices $\mathbf{P}=\mathbf{P}^{T}>0$ and $\mathbf{Q}_{c}=$ $\mathbf{Q}_{c}^{T}>0$ so that the following LMI, 


$$
\left[\begin{array}{cc}
\mathbf{P A}+\mathbf{A}^{T} \mathbf{P}+\mathbf{Q}_{c}+\mathbf{C}^{T} \mathbf{C} & \mathbf{P B} \\
\mathbf{B}^{T} \mathbf{P} & -\gamma^{2}
\end{array}\right]<0,
$$

is feasible, where $\gamma=1 / \delta, \mathbf{C}=\left[\begin{array}{ll}1 & 0\end{array}\right]$, and $\mathbf{A}$ and $\mathbf{B}$ are provided in (45). Then the quadratic form,

$$
V=\zeta^{T} \mathbf{P} \zeta
$$

is a strict Lyapunov function for system (45) and the trajectory reaches the origin in a finite time.

Proof of Theorem 1. Applying the Rayleigh inequality, $V$ is bounded by

$$
\lambda_{\min }(\mathbf{P})\|\zeta\|^{2} \leq V \leq \lambda_{\max }(\mathbf{P})\|\zeta\|^{2},
$$

where $\|\zeta\|^{2}=\left|z_{1}\right|+z_{2}^{2}$ represents the Euclidean norm of $\zeta$. For (48), taking time derivative gives

$$
\dot{V}=\frac{1}{\left|\zeta_{1}\right|}\left[\zeta^{T}\left(\mathbf{P A}+\mathbf{A}^{T} \mathbf{P}\right) \zeta+\zeta^{T} \mathbf{P B} \widetilde{\rho}+\widetilde{\rho} \mathbf{B}^{T} \mathbf{P} \zeta\right]
$$

According to (46), the following inequality is satisfied:

$$
\left|\widetilde{\rho}\left(t, \zeta_{1}\right)\right|^{2} \leq \delta^{2}\left|\zeta_{1}\right|^{2} \leq \delta^{2}\left(\zeta_{1}^{2}+\zeta_{2}^{2}\right),
$$

which guarantees

$$
\zeta^{T} \zeta-\frac{1}{\delta^{2}} \tilde{\rho}\left(t, \zeta_{1}\right)^{2}>0
$$

Hence, (50) can be rewritten as

$$
\begin{aligned}
& \dot{V} \leq \frac{1}{\left|\zeta_{1}\right|}\left[\zeta^{T}\left(\mathbf{P A}+\mathbf{A}^{T} \mathbf{P}\right) \zeta+\zeta^{T} \mathbf{P B} \widetilde{\rho}+\widetilde{\rho} \mathbf{B}^{T} \mathbf{P} \zeta+\zeta^{T} \zeta-\frac{1}{\delta^{2}} \widetilde{\rho}^{2}\right] \\
& =\frac{1}{\left|\zeta_{1}\right|}\left[\zeta^{T}\left(\mathbf{P A}+\mathbf{A}^{T} \mathbf{P}+\mathbf{C}^{T} \mathbf{C}+\mathbf{Q}_{c}-\mathbf{Q}_{c}\right) \zeta+\zeta^{T} \mathbf{P} \mathbf{B} \tilde{\rho}+\tilde{\rho} \mathbf{B}^{T} \mathbf{P} \zeta-\frac{1}{\delta^{2}} \tilde{\rho}^{2}\right] \\
& =-\frac{1}{\left|\zeta_{1}\right|} \zeta^{T} \mathbf{Q}_{c} \zeta+\frac{1}{\left|\zeta_{1}\right|}\left[\begin{array}{l}
\zeta \\
\tilde{\rho}
\end{array}\right]^{T}\left[\begin{array}{cc}
\mathbf{P A}+\mathbf{A}^{T} \mathbf{P}+\mathbf{Q}_{c}+\mathbf{C}^{T} \mathbf{C} & \mathbf{P B} \\
\mathbf{B}^{T} \mathbf{P} & -\gamma^{2}
\end{array}\right]\left[\begin{array}{l}
\zeta \\
\tilde{\rho}
\end{array}\right] .
\end{aligned}
$$

where $\gamma=1 / \delta$.

Based on (47) and (49), (53) can be further simplified to

$$
\dot{V} \leq-\frac{1}{\left|\zeta_{1}\right|} \zeta^{T} \mathbf{Q}_{c} \zeta \leq-\frac{1}{\left|\zeta_{1}\right|} \lambda_{\min }\left(\mathbf{Q}_{c}\right)\|\zeta\|^{2} \leq-\frac{1}{\left|\zeta_{1}\right|} \lambda_{\min }\left(\mathbf{Q}_{c}\right) \frac{V}{\lambda_{\max }(\mathbf{P})} .
$$

Again, from (49), the following inequality can be deduced:

$$
-\frac{1}{\left|\zeta_{1}\right|} \leq-\frac{1}{\|\zeta\|} \leq-\frac{\lambda_{\min }^{1 / 2}(\mathbf{P})}{V^{1 / 2}}
$$

Based on (55), it can be concluded that (54) satisfies

$$
\dot{V} \leq-\alpha V^{1 / 2}, \quad \alpha=\frac{\lambda_{\min }\left(\mathbf{Q}_{c}\right) \lambda_{\min }^{1 / 2}(\mathbf{P})}{\lambda_{\max }(\mathbf{P})} .
$$

For (56), one has

$$
\int_{V(0)}^{V(t)} \frac{\mathrm{d} V}{V^{1 / 2}} \leq-\alpha \int_{0}^{t} \mathrm{~d} t
$$

which implies

$$
V(t) \leq\left(V^{1 / 2}(0)-\frac{\alpha}{2} t\right)^{2} .
$$

Hence, $V(t)$ reaches zero within a finite time described by

$$
t_{f} \leq \frac{2 V^{1 / 2}(0)}{\alpha},
$$

where $V(0)$ is the initial condition of $V(t)$ and $\alpha$ is given by (56). Based on the appropriate gain pair $\left(k_{1}, k_{2}\right)$ selection, the LMI equation (47) can be established. As a result, the finite time stability as shown by (59) can be achieved.

In Theorem 1, we suppose that the LMI (47) is negative definite. Theorem 2 is similar to $[21,22,25]$ and is presented for the feasibility of LMI (48) in Theorem 1.

Theorem 2. Consider the LMI given by (47); there exists a feasible solution $\mathbf{P}, \mathbf{Q}^{c}$ so that the LMI (47) can be established if and only if the parameters $k_{1}$ and $k_{2}$ in $\mathbf{A}$ satisfy

$$
\begin{aligned}
& k_{2}>\delta, \\
& k_{1}^{2}>4 k_{2},
\end{aligned}
$$

or

$$
k_{1}^{2}\left(\frac{1}{2} k_{2}-\frac{1}{16} k_{1}^{2}\right)<\delta^{2}, \quad 4 k_{2}>k_{1}^{2} .
$$

Furthermore, the additional constraints $k_{2} \neq 0$ for (60) and $k_{1} \neq 0$ and $k_{1}^{2} \neq 8 k_{2}$ for (61) must also be satisfied.

Proof of Theorem 2. If the LMI (45) is feasible, then the $\mathscr{L}_{2}$-gain of the following system,

$$
G(s)=\frac{1 / 2}{s^{2}+(1 / 2) k_{1} s+(1 / 2) k_{2}},
$$

must be less than or equal to $\gamma$; that is, 


$$
\max _{\omega}|G(j \omega)|<\gamma=\frac{1}{\delta} \Rightarrow \max _{\omega}|G(j \omega)|^{2}<\frac{1}{\delta^{2}} .
$$

The above statement is the so-called bounded-real condition [40]. In order to find proper $\left(k_{1}, k_{2}\right)$ in $(62)$ so that condition (63) can be satisfied, calculate

$$
|G(j \omega)|^{2}=\frac{1}{\left(k_{2}-2 \omega^{2}\right)^{2}+\left(k_{1} \omega\right)^{2}},
$$

and its derivative

$$
\frac{\mathrm{d}}{\mathrm{d} \omega}|G(j \omega)|^{2}=-\frac{16 \omega\left(\omega^{2}+(1 / 8) k_{1}^{2}-(1 / 2) k_{2}\right)}{\left[\left(k_{2}-2 \omega^{2}\right)^{2}+\left(k_{1} \omega\right)^{2}\right]^{2}} .
$$

The extreme point can be obtained by setting (65) equal to zero. Checking for the second-order sufficient condition $\mathrm{d}^{2} / \mathrm{d} \omega^{2}|G(j \omega)|^{2}$, it can be deduced that $\max _{\omega}|G(j \omega)|$ can be reached, when

$$
\omega= \begin{cases}0, & \text { if } 4 k_{2}-k_{1}^{2}<0 \\ \left(\frac{4 k_{2}-k_{1}^{2}}{8}\right)^{1 / 2}, & \text { if } 4 k_{2}-k_{1}^{2}>0 .\end{cases}
$$

Substituting (66) into (64) yields

$$
\max _{\omega}|G(j \omega)|^{2}= \begin{cases}\frac{1}{k_{2}^{2}}, & \text { if } 4 k_{2}-k_{1}^{2}<0 . \\ \frac{1}{k_{1}^{2}\left((1 / 2) k_{2}-(1 / 16) k_{1}^{2}\right)}, & \text { if } 4 k_{2}-k_{1}^{2}>0 .\end{cases}
$$

Thus, combining (63) and (67) shows that if

$$
\begin{aligned}
& k_{2}>\delta, \\
& k_{1}^{2}>4 k_{2}, \\
& \text { or } \\
& k_{1}^{2}\left(\frac{1}{2} k_{2}-\frac{1}{16} k_{1}^{2}\right)>\delta^{2}, \quad 4 k_{2}>k_{1}^{2},
\end{aligned}
$$

then the LMI (47) is feasible. Moreover, to avoid the singularity of (67), the constraints $k_{2} \neq 0$ for (68) and $k_{1} \neq 0$ and $k_{1}^{2} \neq 8 k_{2}$ for (69) are made.

Remark 1. Notice that system (62) is not a transfer function of system (45); it is the corresponding linear system of LMI (47). More details can be found in [40].

3.2. Controller Design. The control objective is to design a robust control torque $\tau_{c}$ such that the spacecraft can achieve the arbitrary attitude trajectory tracking demands in the presence of time-varying disturbances.

For this reason, let $\mathbf{Q}_{d}=\left[q_{0 d}, \mathbf{q}_{d}^{T}\right]^{T}=\left[q_{0 d}, q_{1 d}, q_{2 d}, q_{3 d}\right]^{T}$ $\in \mathbb{R}^{4}$ be the desired quaternion and let $\boldsymbol{\omega}_{d}=\left[\omega_{x d}, \omega_{y d}\right.$, $\left.\omega_{z d}\right]^{T} \in \mathbb{R}^{3}$ be the desired angular velocity. Define the tracking error vectors $Q_{e}=\left[q_{0 e}, \mathbf{q}_{e}^{T}\right]^{T} \in \mathbb{R}^{4}$ and $\boldsymbol{\omega}_{e}=\left[\omega_{x e}, \omega_{y e}\right.$, $\left.\omega_{z e}\right]^{T} \in \mathbb{R}^{3}$ as follows [36]:

$$
\begin{aligned}
& \mathbf{Q}_{e}=\mathbf{Q}_{d}^{-1} \otimes \mathbf{Q}=\left[\begin{array}{c}
q_{0 e} \\
\mathbf{q}_{e}
\end{array}\right]=\left[\begin{array}{c}
q_{0 d} q_{0}+\mathbf{q}_{d}^{T} \mathbf{q} \\
q_{0 d} \mathbf{q}-q_{0} \mathbf{q}_{d}-\mathbf{q}_{d}^{\times} \mathbf{q}
\end{array}\right], \\
& \boldsymbol{\omega}_{e}=\omega-\omega_{d}=\left[\begin{array}{l}
\omega_{x e} \\
\omega_{y e} \\
\omega_{z e}
\end{array}\right]=\left[\begin{array}{c}
\omega_{x}-\omega_{x d} \\
\omega_{y}-\omega_{y d} \\
\omega_{z}-\omega_{z d}
\end{array}\right],
\end{aligned}
$$

where $\otimes$ represents the quaternion multiplication. Based on (70), the quaternion-based error dynamics of (6), (7), and (25) is given by

$$
\begin{aligned}
& \dot{q}_{0 e}=-\frac{1}{2} \mathbf{q}_{e}^{T} \omega_{e}, \\
& \dot{\mathbf{q}}_{e}=\frac{1}{2}\left(q_{0 e} \mathbf{I}_{3}+\mathbf{q}_{e}^{\times}\right) \omega_{e}, \\
& \dot{\omega}_{e}=\mathbf{J}_{e q}^{-1}\left[-\boldsymbol{\tau}_{c}+\mathbf{d}-\omega^{\times}\left(\mathbf{J}_{e q} \omega+\Gamma \mathbf{J}_{m} \mathbf{\Omega}\right)\right]-\dot{\omega}_{d} .
\end{aligned}
$$

Select the sliding surface as

$$
\mathbf{S}=\boldsymbol{\omega}_{e}+\lambda \mathbf{q}_{e},
$$

where $\mathbf{S}=\left[s_{1}, s_{2}, s_{3}\right]^{T} \in \mathbb{R}^{3} ; \lambda \in \mathbb{R}^{1}$ is a positive parameter to be designed.

Suppose that the sliding motion is fulfilled in a finite time $t=t_{f}$; it gives

$$
\mathbf{S}=\dot{\mathbf{S}}=\mathbf{0}, \quad \forall t \geq t_{f} .
$$

From (71), (72), (74), and (75), the nonlinear reducedorder dynamics can be obtained:

$$
\begin{aligned}
\dot{q}_{0 e} & =-\frac{1}{2} \mathbf{q}_{e}^{T} \omega_{e} \\
& =-\frac{1}{2} \mathbf{q}_{e}^{T}\left(-\lambda \mathbf{q}_{e}\right) \\
& =\frac{1}{2} \lambda \mathbf{q}_{e}^{T} \mathbf{q}_{e}, \quad \forall t \geq t_{f}, \\
\dot{\mathbf{q}}_{e} & =\frac{1}{2}\left(q_{0 e} \mathbf{I}_{3}+\mathbf{q}_{e}^{\times}\right)\left(-\lambda \mathbf{q}_{e}\right) \\
& =-\frac{1}{2} \lambda q_{0 e} \mathbf{q}_{e}, \quad \forall t \geq t_{f} .
\end{aligned}
$$

The term "reduced-order" means that the system error dynamics described by (71)-(73 with order 7 "reduced" to subsystem (76) and (77) with order 4 . In order to analyze the stability of the reduced-order dynamics, apply the identity of unit quaternion:

$$
q_{0 e}^{2}+\mathbf{q}_{e}^{T} \mathbf{q}_{e}=1
$$

Equation (76) can be decoupled as

$$
\dot{q}_{0 e}=\frac{1}{2} \lambda\left(1-q_{0 e}^{2}\right), \quad \forall t \geq t_{f} .
$$

Then, consider the following variable transformation: 


$$
q_{0 e}(t)=1+\frac{1}{y(t)}
$$

which implies

$$
\dot{q}_{0 e}(t)=-\frac{\dot{y}(t)}{y^{2}}
$$

Substituting (79) yields

$$
-\frac{\dot{y}(t)}{y^{2}}=\frac{1}{2} \lambda\left[1-\left(1+\frac{1}{y(t)}\right)^{2}\right]=\frac{1}{2} \lambda\left(-\frac{2}{y}-\frac{1}{y^{2}}\right),
$$

which implies

$$
\dot{y}-\lambda y=\frac{1}{2} \lambda
$$

Clearly, equation (83) is a linear ordinary differential equation. Its solution is given by

$$
y(t)=C e^{\lambda t}-\frac{1}{2}
$$

where $C$ is an integration constant. Apply the inverse mapping from (80); we get

$$
q_{0 e}(t)=1+\frac{1}{-(1 / 2)+C e^{\lambda t}}
$$

Let $q_{0 e}(0)$ be the initial condition; we have

$$
C=\frac{1}{2}+\frac{1}{q_{0 e}(0)-1}
$$

Hence, the analytic solution of (79) can be obtained:

$$
q_{0 e}(t)=1+\frac{2\left[q_{0 e}\left(t_{f}\right)-1\right]}{-q_{0 e}\left(t_{f}\right)+1+\left[q_{0 e}\left(t_{f}\right)+1\right] e^{\lambda\left(t-t_{f}\right)}}, \quad \forall t \geq t_{f}
$$

Observing (87), it can be deduced that (i) the singularity occurs as an improper sliding gain $\lambda$ is chosen such that $-q_{0 e}\left(t_{f}\right)+1+\left[q_{0 e}\left(t_{f}\right)+1\right] e^{\lambda\left(t-t_{f}\right)}=0, \forall t \geq t_{f}$. Thus, the sliding gain $\lambda>0$ is designed so that $-q_{0 e}\left(t_{f}\right)+1+\left[q_{0 e}\left(t_{f}\right)+1\right] e^{\lambda\left(t-t_{f}\right)} \neq 0, \forall t \geq t_{f}$. $q_{0 e} \longrightarrow+1$ as $t \longrightarrow \infty$ from (87) and $\mathbf{q}_{e} \longrightarrow 0$ as $t \longrightarrow \infty$ from the identity of the unit quaternion (78). Observing (70), it can be found that $q_{0 e} \longrightarrow 1$ and $\mathbf{q}_{e} \longrightarrow 0$ imply $\mathbf{q}_{0} \longrightarrow \mathbf{q}_{0 d}$ and $\overrightarrow{\mathbf{q}} \longrightarrow \overrightarrow{\mathbf{q}}_{d}$. That is, the nonlinear reducedorder dynamics (76) and (77) are asymptotically stable. It is different and more outstanding than $[30,35,36]$; the assumption that $q_{0 e} \neq 0$ is not made. In the following, how to enter the sliding mode in a finite time in the presence of external disturbances by means of the robust control law will be discussed.

In order to introduce $\tau_{c}$, taking the time derivative about (74) yields

$$
\begin{aligned}
\dot{\mathbf{S}} & =\dot{\omega}_{e}+\lambda \dot{\mathbf{q}}_{e} \\
& =\mathbf{J}_{e q}^{-1}\left[-\boldsymbol{\tau}_{c}+\mathbf{d}-\omega^{\times}\left(\mathbf{J}_{e q} \omega+\Gamma \mathbf{J}_{m} \boldsymbol{\Omega}\right)\right]+\boldsymbol{\xi},
\end{aligned}
$$

where

$$
\boldsymbol{\xi}=-\dot{\boldsymbol{\omega}}_{d}+0.5 \lambda\left(q_{0 e} \mathbf{I}_{3}+\mathbf{q}_{e}^{\times}\right) \boldsymbol{\omega}_{e} .
$$

Based on the super-twisting sliding mode algorithm [21], the following robust control law is designed:

$$
\begin{aligned}
\boldsymbol{\tau}_{c 0} & =\omega^{\times}\left(\mathbf{J}_{e q} \omega+\Gamma \mathbf{J}_{m} \mathbf{\Omega}\right)-\mathbf{J}_{e q} \xi \\
\boldsymbol{\tau}_{c N} & =-\mathbf{J}_{e q}\left(\mathbf{K}_{1} \frac{\mathbf{S}}{\|\mathbf{S}\|^{1 / 2}}+\mathbf{K}_{2} \int_{0}^{t} \operatorname{sign}(\mathbf{S}(\tau)) \mathrm{d} \tau\right), \\
\boldsymbol{\tau}_{c} & =-\left(\boldsymbol{\tau}_{c 0}+\boldsymbol{\tau}_{c N}\right),
\end{aligned}
$$

in which the gain matrices are denoted as

$$
\begin{aligned}
& \mathbf{K}_{1}=\operatorname{diag}\left(\left[\begin{array}{lll}
k_{11} & k_{12} & k_{13}
\end{array}\right]\right), \\
& \mathbf{K}_{2}=\operatorname{diag}\left(\left[\begin{array}{lll}
k_{21} & k_{22} & k_{23}
\end{array}\right]\right) .
\end{aligned}
$$

Substituting (90) into (88) yields the closed-loop sliding dynamics:

$$
\dot{\mathbf{S}}=-\mathbf{K}_{1} \frac{\mathbf{S}}{\|\mathbf{S}\|^{1 / 2}}-\mathbf{K}_{2} \int_{0}^{t} \operatorname{sign}(\mathbf{S}(\boldsymbol{\tau})) \mathrm{d} \tau+\mathbf{D},
$$

where $\mathbf{D}=\mathbf{J}_{e q}^{-1} \mathbf{d}=\left[D_{1}, D_{2}, D_{3}\right]^{T} \in \mathbb{R}^{3}$ and $\operatorname{sign}(\mathbf{S}) \in \mathbb{R}^{3}$ is a sign function defined as follows:

$$
\begin{aligned}
& \operatorname{sign}\left(s_{i}\right)= \begin{cases}1, & \text { if } s_{i}>0, \\
-1, & \text { if } s_{i}<0,\end{cases} \\
& \operatorname{sign}\left(s_{i}\right) \in[-1,1], \quad \text { if } s_{i}=0,(i=1,2,3 .) .
\end{aligned}
$$

According to the stability criteria derived from Theorems 1 and 2, the following gains are chosen:

$$
\begin{aligned}
& k_{2 i}>\delta_{i}, \\
& k_{1 i}>\sqrt{4 k_{2 i}},
\end{aligned}
$$

where $\delta_{i}=\sup \left(\dot{D}_{i}\right), i=1,2,3$. 


\section{Spacecraft Reference Command Generation}

4.1. Reference Quaternion Generation from TNB Frame. In this section, we are going to illustrate how to obtain the reference quaternion from the TNB frame. The procedures are as follows:

(1) Reference orbital trajectory: Consider the governing equations of the orbital motion [41]:

$$
\ddot{\mathbf{r}}=-\frac{\mu}{r^{3}} \mathbf{r}
$$

where $r=[X, Y, Z]^{T} \in \mathbb{R}^{3}$ is the position vector and its magnitude is $r=\|\mathbf{r}\| ; \mu$ is the gravitational parameter. The reference orbital trajectory can be obtained by integrating (95) in a given initial condition.

(2) Construction of the TNB Frame: According to geometry kinematics, the TNB frame can be constructed by the trajectory information. Let $\mathbf{v}=\dot{\mathbf{r}}$ be the velocity vector and let $\mathbf{a}=\ddot{\mathbf{r}}$ be the acceleration vector. The unit tangent vector can be computed by

$$
\widehat{\mathbf{e}}_{t}=\frac{\mathbf{v}}{\|\mathbf{v}\|} \text {. }
$$

The unit normal vector can be obtained by

$$
\widehat{\mathbf{e}}_{n}=\frac{\mathbf{a}_{n}}{\left\|\mathbf{a}_{n}\right\|} .
$$

where $\mathbf{a}_{n}=\mathbf{a}-\mathbf{a}_{t}$ is the normal acceleration and $\mathbf{a}_{t}=$ $\left(\mathbf{a} \cdot \widehat{\mathbf{e}}_{t}\right) \widehat{\mathbf{e}}_{t}$ is the tangential acceleration. Based on the definition of TNB frame, the binormal vector is

$$
\widehat{\mathbf{e}}_{b}=\widehat{\mathbf{e}}_{t} \times \widehat{\mathbf{e}}_{n} .
$$

(3) Reference Quaternion: Based on the rotational property, the direction cosine matrix (DCM) can be composed by $\widehat{\mathbf{e}}_{t}, \widehat{\mathbf{e}}_{n}$, and $\widehat{\mathbf{e}}_{b}$; that is,

$$
\mathbf{C}^{v}=\left[\widehat{\mathbf{e}}_{t}: \widehat{\mathbf{e}}_{n}: \widehat{\mathbf{e}}_{b}\right]
$$

Applying the quaternion kinematics, the reference quaternion is

$$
\begin{aligned}
& q_{0 d}=\frac{1}{2}\left(1+\mathbf{C}_{11}^{v}+\mathbf{C}_{22}^{v}+\mathbf{C}_{33}^{v}\right)^{1 / 2}, q_{1 d}=\frac{1}{4 q_{0}}\left(\mathbf{C}_{32}^{v}-\mathbf{C}_{23}^{v}\right), \\
& q_{2 d}=\frac{1}{4 q_{0}}\left(\mathbf{C}_{13}^{v}-\mathbf{C}_{31}^{v}\right), q_{3 d}=\frac{1}{4 q_{0}}\left(\mathbf{C}_{21}^{v}-\mathbf{C}_{12}^{v}\right) .
\end{aligned}
$$

4.2. Reference Angular Velocity Generation. To generate a feasible command trajectory, the following property is proposed [35].
Property 1. The matrix E(Q) defined in (9) has the following properties:

$$
\begin{aligned}
\mathbf{E}^{T}(\mathbf{Q}) \mathbf{E}(\mathbf{Q}) & =\mathbf{I}_{3}, \\
\frac{\mathrm{d}}{\mathrm{d} t}\left[\mathbf{E}^{T}(\mathbf{Q}) \dot{\mathbf{Q}}\right] & =\mathbf{E}^{T}(\mathbf{Q}) \ddot{\mathbf{Q}}
\end{aligned}
$$

By using Property 1, from (8), the angular velocity and its time derivative can be expressed as

$$
\begin{aligned}
& \boldsymbol{\omega}=2 \mathbf{E}^{T}(\mathbf{Q}) \dot{\mathbf{Q}}, \\
& \dot{\boldsymbol{\omega}}=2 \mathbf{E}^{T}(\mathbf{Q}) \ddot{\mathbf{Q}}
\end{aligned}
$$

Hence, the formula of the desired angular velocity $\omega_{d}$ associated with the desired quaternion is

$$
\begin{aligned}
& \boldsymbol{\omega}_{d}=2 \mathbf{E}^{T}\left(\mathbf{Q}_{d}\right) \dot{\mathbf{Q}}_{d}, \\
& \dot{\boldsymbol{\omega}}_{d}=2 \mathbf{E}^{T}\left(\mathbf{Q}_{d}\right) \ddot{\mathbf{Q}}_{d}
\end{aligned}
$$

where

$$
\mathbf{E}\left(\mathbf{Q}_{d}\right)=\left[\begin{array}{ccc}
-q_{1 d} & -q_{2 d} & -q_{3 d} \\
q_{0 d} & -q_{3 d} & q_{2 d} \\
q_{3 d} & q_{0 d} & -q_{1 d} \\
-q_{2 d} & q_{1 d} & q_{0 d}
\end{array}\right]
$$

\section{Numerical Simulation}

According to [42], the numerical data are considered and summarized as follows.

(i) Moment of inertia of the spacecraft and reaction wheels is as follows:

$$
\begin{aligned}
\mathbf{J} & =\left[\begin{array}{ccc}
35 & 3 & -1.5 \\
3 & 28 & 2 \\
-1.5 & 2 & 30
\end{array}\right], \\
\mathbf{J}_{w} & =\left[\begin{array}{ccc}
0.126 & 0 & 0 \\
0 & 0.063 & 0 \\
0 & 0 & 0.063
\end{array}\right](N-\mathrm{m}) .
\end{aligned}
$$

(ii) The external disturbance is chosen as

$$
\mathbf{d}(t)=0.005[\sin 0.8 t \quad \cos 0.5 t \quad \cos 0.3 t]^{T}(\mathrm{~N}-\mathrm{m}) .
$$

(iii) Initial conditions are the following [41]: $\mathbf{r}(0)=$ $[8000,0,6000]^{T} \quad(\mathrm{~km}) ; \dot{\mathbf{r}}(0)=[0,7,0]^{T} \quad(\mathrm{~km} / \mathrm{s}) ;$ $\boldsymbol{\omega}(0)=[7,-8,-7]^{T} \quad($ degree $/$ sec $) ; \quad \mathbf{Q}(0)=[0.999$, $0.017,-0.035,-0.026]^{T}$; and gravitational parameter $\mu=3.987 \times 10^{5}\left(\mathrm{~N}-\mathrm{km}^{2} / \mathrm{kg}\right)$. 


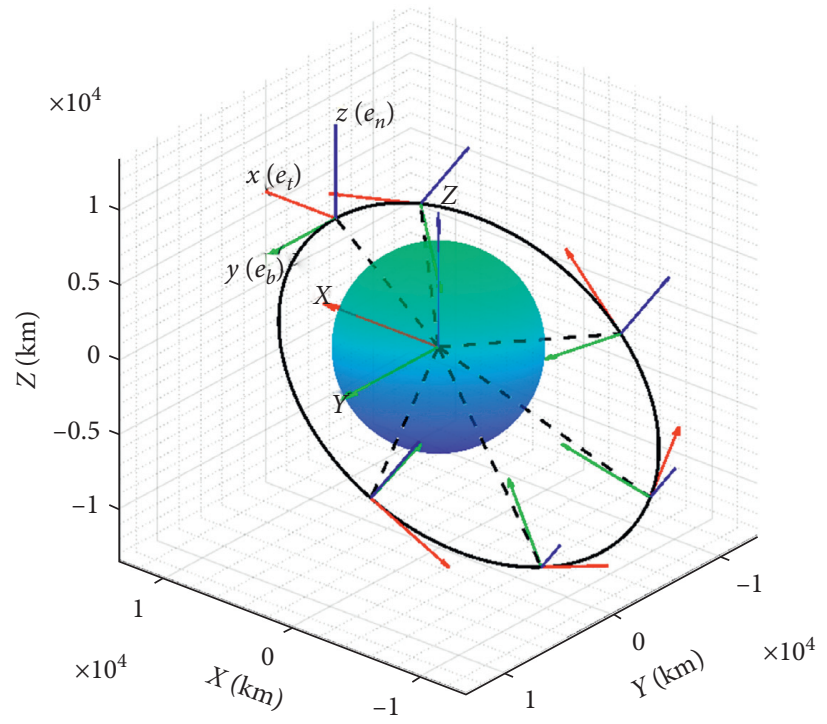

Figure 4: Spacecraft 3D trajectory.

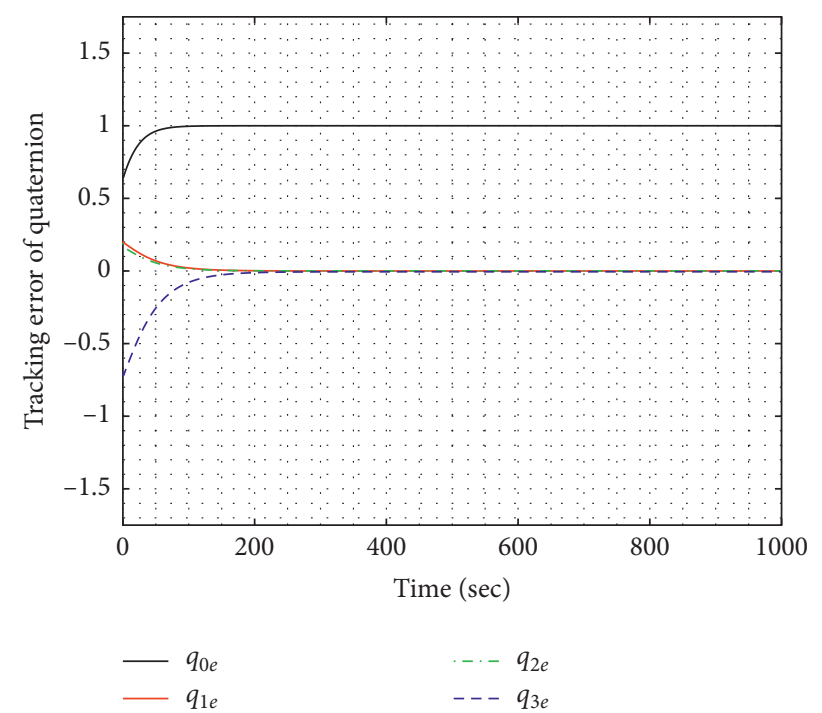

FIGURE 5: Quaternion tracking error.

(iv) The control gains are

$$
\begin{aligned}
\mathbf{K}_{1} & =\operatorname{diag}\left(\left[\begin{array}{lll}
0.547 & 0.547 & 0.547
\end{array}\right]\right), \\
\mathbf{K}_{2} & =\operatorname{diag}\left(\left[\begin{array}{lll}
0.05 & 0.05 & 0.05
\end{array}\right]\right), \\
\lambda & =0.05
\end{aligned}
$$

The sampling rate is $400 \mathrm{~Hz}$. Total time span is 4.17 hours. The simulation results are shown in Figures 4-7. For convenience, only the transition response is shown.
With regard to Figure 4, it is demonstrated that the spacecraft tracks the reference TNB attitude trajectory successfully. The attitude of the spacecraft with respect to the global frame $X Y Z$ is represented as $x y z$. The response of quaternion tracking errors is shown in Figure 5. It can verify the derivation of analytic solution (87) that the scalar component $q_{0 e} \longrightarrow 1$ and the vector component $\mathbf{q}_{e} \longrightarrow \mathbf{0}$ as $t \longrightarrow \infty$ and they satisfy the constraint $q_{0 e}+\mathbf{q}_{e}^{T} \mathbf{q}_{e}=1$. Figure 6 illustrates the response of the sliding variable. It can be found that the converging speed of the sliding variables is fast even though the spacecraft is under the environment with the time-varying external disturbances. The evolution 

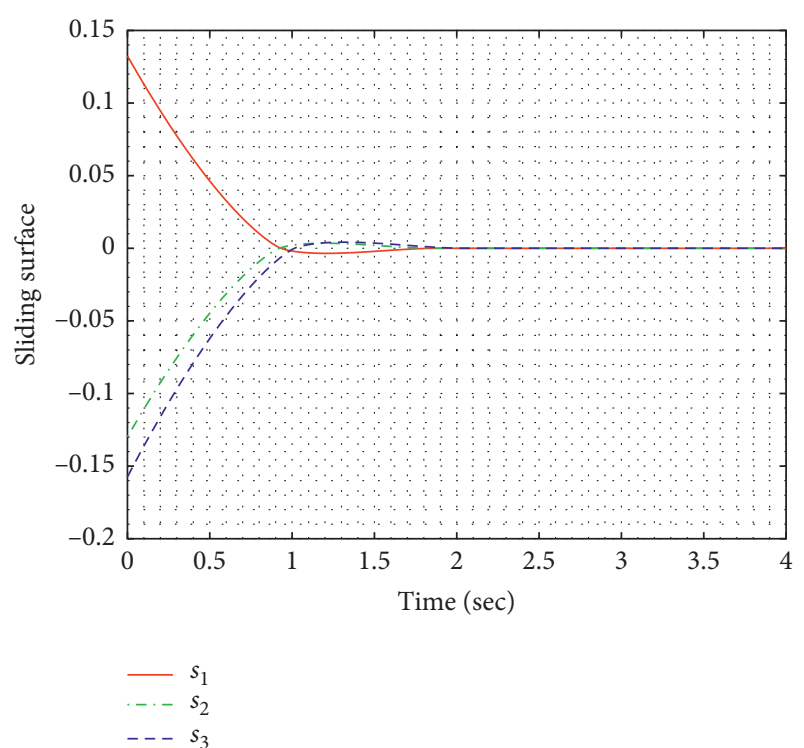

FIGURE 6: Sliding surface.

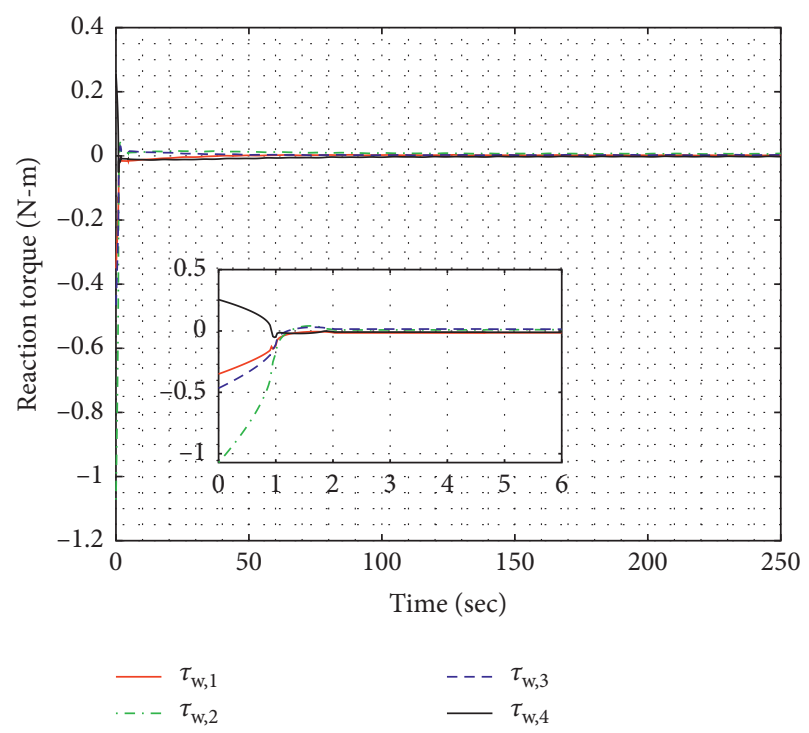

Figure 7: Evolution of reaction torque.

of the reaction torques is shown in Figure 7 . It can be seen that the control signal is smooth and there is no chattering phenomenon. We can deduce that the controller based on the super-twisting sliding algorithm can be realized in the spacecraft attitude control problem.

\section{Conclusion}

In this study, the attitude dynamics based on the redundant reaction wheels configuration of the spacecraft is derived. To achieve full degree of freedom attitude tracking control, the quaternion kinematics is introduced. For practical realization purpose, the nonsquare FDM is reformulated as a square, invertible matrix by means of solving a static optimization problem. The robust, continuous supertwisting sliding mode algorithm is adopted in the attitude controller design to guarantee robust performance in the presence of exogenous disturbances. Furthermore, the corresponding finite time stability based on the LMI is provided in the sense of Lyapunov. Asymptotic stability of the nonlinear reduced-order dynamics is proven by means of an analytic solution without imposing any limitation in quaternion. In a space mission with this scenario, the TNB command generation strategy is presented. Finally, the simulation results verify that the spacecraft can achieve the arbitrary attitude trajectory tracking demands in the presence of the time-varying external disturbances.

\section{Data Availability}

No data or software is included in the submission.

\section{Conflicts of Interest}

The authors declare no conflicts of interest.

\section{Acknowledgments}

This work was supported by the Ministry of Science and Technology under Grant nos. MOST 107-2221-E-006-114MY3 and MOST 108-2923-E-006-005-MY3.

\section{References}

[1] Z. Feng and J. Fei, "Design and analysis of adaptive supertwisting sliding mode control for a microgyroscope," PloS One, vol. 13, no. 1, 2018.

[2] Z. Wang, Q. Li, and S. Li, “Adaptive integral-type terminal sliding mode fault tolerant control for spacecraft attitude tracking," IEEE Access, vol. 7, pp. 35195-35207, 2019.

[3] Y. Zhao, J. Wang, F. Yan, and Y. Shen, "Adaptive sliding mode fault-tolerant control for type-2 fuzzy systems with distributed delays," Information Sciences, vol. 473, pp. 227-238, 2019.

[4] G. Sun, L. Wu, Z. Kuang, Z. Ma, and J. Liu, "Practical tracking control of linear motor via fractional-order sliding mode," Automatica, vol. 94, pp. 221-235, 2018.

[5] L. Wang and Z. Song, "Continuous fixed-time sliding mode attitude controller design for rigid-body spacecraft," IEEE Access, vol. 8, pp. 105399-105410, 2020.

[6] L. Luque-Vega, B. Castillo-Toledo, and A. G. Loukianov, "Robust block second order sliding mode control for a quadrotor," Journal of the Franklin Institute, vol. 349, no. 2, pp. 719-739, 2012.

[7] F. Chen, R. Jiang, K. Zhang, B. Jiang, and G. Tao, "Robust backstepping sliding-mode control and observer-based fault estimation for a quadrotor uav," IEEE Transactions on Industrial Electronics, vol. 63, no. 8, pp. 5044-5056, 2016.

[8] D. Almakhles, "Robust backstepping sliding mode control for a quadrotor trajectory tracking application," IEEE Access, vol. 63, 2019.

[9] F. Bayat, "Model predictive sliding control for finite-time three-axis spacecraft attitude tracking," IEEE Transactions on Industrial Electronics, vol. 66, no. 10, pp. 7986-7996, 2018. 
[10] Y. Shtessel, C. Edwards, L. Fridman, and A. Levant, "Sliding mode control and observation," Springer, vol. 6, 2014.

[11] Y. B. Shtessel, J. A. Moreno, F. Plestan, L. M. Fridman, and S. Alexander, Super-twisting adaptive sliding mode control: a lyapunov design, 2010.

[12] Y. Shtessel, F. Plestan, and M. Taleb, "Lyapunov design of adaptive super-twisting controller applied to a pneumatic actuator," IFAC Proceedings Volumes, vol. 44, no. 1, pp. 3051-3056, 2011.

[13] Y. Shtessel, M. Taleb, and F. Plestan, "A novel adaptive-gain supertwisting sliding mode controller: methodology and application," Automatica, vol. 48, no. 5, pp. 759-769, 2012.

[14] V. I. Utkin and A. S. Poznyak, "Adaptive sliding mode control with application to super-twist algorithm: equivalent control method," Automatica, vol. 49, no. 1, pp. 39-47, 2013.

[15] K. Lu and Y. Xia, "Finite-time attitude control for rigid spacecraft-based on adaptive super-twisting algorithm," IET Control Theory \& Applications, vol. 8, no. 15, pp. 1465-1477, 2014.

[16] C.-C. Peng and C.-L. Chen, "Robust chaotic control of lorenz system by backstepping design," Chaos, Solitons \& Fractals, vol. 37, no. 2, pp. 598-608, 2008.

[17] C.-L. Chen, C. C. Peng, and H.-T. Yau, "High-order sliding mode controller with backstepping design for aeroelastic systems," Communications in Nonlinear Science and $\mathrm{Nu}$ merical Simulation, vol. 17, no. 4, pp. 1813-1823, 2012.

[18] L.-H. Chen and C.-C. Peng, "Extended backstepping sliding controller design for chattering attenuation and its application for servo motor control," Applied Sciences, vol. 7, no. 3, p. $220,2017$.

[19] A. Levant, "Sliding order and sliding accuracy in sliding mode control," International Journal of Control, vol. 58, no. 6, pp. 1247-1263, 1993.

[20] J. A. Moreno and M. Osorio, A lyapunov approach to secondorder sliding mode controllers and observers, 2008.

[21] A. Dávila, J. A. Moreno, and L. Fridman, "Optimal lyapunov function selection for reaching time estimation of super twisting algorithm," in Proceedings of the 48h IEEE Conference on Decision and Control (CDC) Held Jointly with 2009 28th Chinese Control Conference, pp. 8405-8410, IEEE, London, UK, 2009.

[22] J. A. Moreno, "A linear framework for the robust stability analysis of a generalized super-twisting algorithm," in Proceedings of the 2009 6th International Conference on Electrical Engineering, Computing Science and Automatic Control (CCE), IEEE, London, UK, 2009.

[23] J. A. Moreno and M. Osorio, "Strict lyapunov functions for the super-twisting algorithm," IEEE Transactions on Automatic Control, vol. 57, no. 4, pp. 1035-1040, 2012.

[24] V. Utkin, "On convergence time and disturbance rejection of super-twisting control," IEEE Transactions on Automatic Control, vol. 58, no. 8, 2013.

[25] L. Derafa, A. Benallegue, and L. Fridman, "Super twisting control algorithm for the attitude tracking of a four rotors uav," Journal of the Franklin Institute, vol. 349, no. 2, pp. 685-699, 2012.

[26] L. Derafa, "Quadcopter robust adaptive second order sliding mode control based on pid sliding surface," IEEE Access, vol. 6, pp. 66850-66860, 2018.

[27] A. Chalanga, S. Kamal, L. M. Fridman, B. Bandyopadhyay , and J. A. Moreno, "Implementation of super-twisting control: super-twisting and higher order sliding-mode observer-based approaches," IEEE Transactions on Industrial Electronics, vol. 63, no. 6, pp. 3677-3685, 2016.
[28] M. Zhang, J. Huang, and F. Chen, Super twisting control algorithm for velocity control of mobile wheeled inverted pendulum systems, 2018.

[29] S. Nomura, S. Ikari, and S. Nakasuka, "Three-axis attitude maneuver of spacecraft by reaction wheels with rotation speed constraints," IFAC-PapersOnLine, vol. 49, no. 17, pp. 130-134, 2016.

[30] M. Navabi and M. R. Hosseini, Spacecraft quaternion based attitude input-output feedback linearization control using reaction wheels, 2017.

[31] M. Navabi, "Globally asymptotic three-axis attitude control for a two-wheeled small satellite," Acta Astronautica, vol. 157, pp. 17-28, 2019.

[32] M. Muehlebach and R. D'Andrea, "Nonlinear analysis and control of a reaction-wheel-based 3-d inverted pendulum," IEEE Transactions on Control Systems Technology, vol. 25, no. 1, pp. 235-246, 2016.

[33] D. Gutiérrez-Oribio, Á. Mercado-Uribe, J. A. Moreno, and L. Fridman, "Reaction wheel pendulum control using fourthorder discontinuous integral algorithm," International Journal of Robust and Nonlinear Control, vol. 31, 2020.

[34] N. A. Chaturvedi, K. Amit, and N. H. McClamroch, "Rigidbody attitude control," IEEE Control Systems Magazine, vol. 31, no. 3, pp. 30-51, 2011.

[35] S.-C. Lo and Y.-P. Chen, "Smooth sliding-mode control for spacecraft attitude tracking maneuvers," Journal of Guidance, Control, and Dynamics, vol. 18, no. 6, pp. 1345-1349, 1995.

[36] S. Wu, G. Radice, Y. Gao, and Z. Sun, "Quaternion-based finite time control for spacecraft attitude tracking," Acta Astronautica, vol. 69, no. 1-2, pp. 48-58, 2011.

[37] K. Ibrahim, Comparison and analysis of attitude control systems of a satellite using reaction wheel actuators, 2012.

[38] Z. Ismail and R. Varatharajoo, "A study of reaction wheel configurations for a 3-axis satellite attitude control," Advances in Space Research, vol. 45, no. 6, pp. 750-759, 2010.

[39] F. L. Lewis, D. Vrabie, and V. L. Syrmos, Optimal Control, John Wiley \& Sons, New York, NY, USA, 2012.

[40] S. Boyd, L. El Ghaoui, E. Feron, and V. Balakrishnan, Linear matrix inequalities in system and control theory, 1994.

[41] H. D. Curtis, Orbital Mechanics for Engineering Students, Butterworth-Heinemann, New York, NY, USA, 2013.

[42] A. Zhang, J. Ni, and H. Reza Karimi, "Reaction wheel installation deviation compensation for overactuated spacecraft with finite-time attitude control," Mathematical Problems in Engineering, vol. 4, 2013. 